\title{
The Role of Diagnostic Management in COVID-19 Screening of Asymptomatic Patients Admitted for Elective Surgery
}

\author{
JAROSLAW CWALINSKI ${ }^{1}$, AGNIESZKA CWALINSKA ${ }^{2}$, JACEK PASZKOWSKI ${ }^{1}$, \\ TOMASZ BANASIEWICZ ${ }^{1}$ and JACEK HERMANN ${ }^{1}$ \\ ${ }^{1}$ Department of General, Endocrinological Surgery and Gastroenterological Oncology, \\ Poznan University of Medical Sciences, Poznan, Poland; \\ ${ }^{2}$ Department of Infectious Diseases and Child Neurology, \\ University of Medical Sciences in Poznan, Poznan, Poland
}

\begin{abstract}
Background: Due to the high risk of COVID-19 transmission by asymptomatic patients, the aim of this study was to evaluate chest computed tomography $(C T)$ and blood differential test as an additional COVID-19 screening tool for patients undergoing elective or urgent surgery. Patients and Methods: The preoperative assessment of 118 patients hospitalized from June to July 2020 included real-time reverse transcriptase polymerase chain reaction RNA test before elective surgery or rapid antigen test in emergency patients. The diagnostics were supplemented by chest $C T$ and a complete blood count with a blood smear in all patients. Results: None of the hospitalized patients had molecular, serological or radiographic symptoms of COVID-19 infection. The chest CT revealed non-COVID-19 pathologies in a total of 48 patients. Leukocytosis and lymphopenia were typical of emergency patients. Conclusion: Routine chest CT scans have no benefit in screening for potential COVID-19 changes in asymptomatic patients. Blood differential tests are readily available, which makes them more helpful in COVID-19 screening.
\end{abstract}

Infection with the new coronavirus COVID-19 has become a global problem. An infectious disease that spreads well beyond a local epidemic to become a major global epidemiological and socio-economic challenge has not been observed for decades.

This article is freely accessible online.

Correspondence to: Jarosław Cwalinski, Department of General, Endocrinological Surgery and Gastroenterological Oncology, Poznan University of Medical Sciences, Przbyszewskiego 49, 60355 Poznan, Poland. Tel.: +48 618691275, Mobile: +48 505487141, e-mail: cwalinski.jaroslaw@spsk2.pl

Key Words: COVID-19 screening, preoperative management, COVID-19 before surgery, exclusion criteria, COVID-19 pandemic, SARS-CoV-2 prevention.
Initially, healthcare centers had first focused on control of infection transmission and treatment. Recently, however, as the pandemic infection rate continues to decline in most countries, healthcare centers have concentrated on streamlining their work organization, for surgical wards in particular. Hence, it is crucial to define such a system of preoperative preparation and patient qualification as to minimize the perioperative risk of virus transmission. The development of a diagnostic algorithm capable of identifying infected patients who are asymptomatic is of central importance. The actual diagnostic gold standard includes rapid antigen test and in the case of high-risk patients, real-time reverse transcriptase polymerase chain reaction (RTPCR). However, there is still a window during which infection remains latent. Therefore, in the first months of the pandemic, chest computed tomography (CT) and blood smear were considered complementary tests in the diagnosis of COVID-19 infection (1). The aim of this study was to evaluate chest CT and the complete blood count with differential as diagnostic tests for COVID-19 infection in low-risk patients who qualified for elective surgery.

\section{Patients and Methods}

The study involved a total of 118 patients who were operated on in the Authors' surgery clinic between June and July 2020. Of this number, 94 admissions were elective and 24 were emergency. General anesthesia was performed in 104 cases, of which 83 required endotracheal tube. All patients were evaluated for potential risk of SARS-CoV-2 infection based on their history and clinical symptoms prior to admission. A routine RT-PCR RNA test was performed on all patients who qualified for elective surgery, whereas a rapid antigen test only was carried out in patients admitted for emergency reasons. In order to detect disease latency in the time window, the diagnostics were extended by chest CT scan and a complete blood count with a blood smear. Radiographic features of interstitial pneumonia and a decrease in the level of lymphocytes below $1,1 \times 10^{9} / 1$ were assumed as being significant for infection. 
Table I. Breakdown of chest computed tomography (CT) results.

\begin{tabular}{lc}
\hline Chest CT result & $\mathrm{N}(\%)$ \\
\hline Without abnormality & $70(59.3 \%)$ \\
With abnormality & $48(40.7 \%)$ \\
Nodular changes & 20 \\
Focal fibrosis & 16 \\
Inflammatory changes (non-COVID) & 5 \\
Hydrothorax & 5 \\
Metastatic changes & 1 \\
Chest/lung trauma & 1 \\
\hline
\end{tabular}

Statistical analysis. The results obtained in the CT examination and morphology were subjected to statistical analysis performed by Statistica software version 6.0 PL (Statsoft, Tulsa, OK, USA). The data were evaluated with Student's $t$-test and Fisher's exact test. A $p$-value of 0.05 was considered statistically significant.

\section{Results}

None of the hospitalized patients presented with symptoms that would point to a potential COVID19 infection. RTPCR RNA or antigen tests were also negative. None of the chest CT scans were characteristic for changes due to COVID19. The chest CT scan was positive for nonCOVID19-related changes in 48 cases, which included among others 20 with nodular abnormalities, five with inflammatory changes and five with hydrothorax (Table I). A lymphocyte count below $1,1 \times 10^{9} / 1$ was detected in a total of 12 patients, of which six cases were elective and six were emergency surgeries (Table II).

\section{Discussion}

Strategies of preoperative diagnostic screening for a potential COVID19 infection in patients qualifying for an elective surgery are under dispute. Qualifying an infected patient for an elective surgery who presents very few and weak symptoms or is asymptomatic is of the greatest concern because of the risk of transmission of infection to other patients or personnel. One of the solutions adopted by countries with a relatively high SARS-CoV-2 infection rate is screening with RT-PCR test at admission, irrespectively of admission type (emergency or elective) $(2,3)$.

RT-PCR testing of the material from the nasopharyngeal swab is a first-line diagnostic technique for COVID-19 infection (3-5). The technique is useful for infection disease monitoring and as a criterion for hospital discharge or transfer. However, a high false-negative rate is common, among others, due to the time of sample collection and disease course mismatch. Therefore, two consecutive RTPCR tests over 24-72 hours are recommended to minimize the false-negative rate (1).
Table II. Leucocyte, lymphocyte and chest computed tomography (CT) status according to type of admission.

\begin{tabular}{lccc}
\hline Parameter & $\begin{array}{c}\text { Emergency } \\
\text { admission } \\
(\mathrm{N}=24)\end{array}$ & $\begin{array}{c}\text { Elective } \\
\text { admission } \\
(\mathrm{N}=94)\end{array}$ & $p$-Value \\
\hline Leucocyte count $>11 \times 10^{9} / 1$ & $9(37.5 \%)$ & $7(7.4 \%)$ & 0.0006 \\
Lymphocyte count $<1.1 \times 10^{9} / 1$ & $6(25 \%)$ & $6(6.4 \%)$ & 0.0154 \\
Chest CT abnormality & $13(54.2 \%)$ & $35(38.9 \%)$ & 0.1642 \\
\hline
\end{tabular}

Some countries have adopted an alternative strategy, which complements the RT-PCR test with a chest X-ray or a chest CT scan to verify the patient's clinical status $(2,6)$. Blood biomarkers such as $\mathrm{C}$-reactive protein, lymphocyte count, low procalcitonin, elevated interleukin-6 and -10 levels may also prove helpful $(1,5)$. Yet Polish guidelines restrict RT-PCR test to symptomatic patients only and recommend chest imaging with X-ray as a modality of first choice in cases of confirmed infection and symptoms suggesting lung involvement (5).

The lack of unequivocal guidelines for a preoperative SARS-Cov-2 prophylactic strategy for low-risk patients have led our team of anesthesiologists, surgeons and epidemiologists to define, in the first months of the pandemic, measures for a rational evaluation of perioperative infection risk. Hence, this study presents the results of chest $\mathrm{CT}$ and the blood differential test performed routinely for all patients admitted to our clinic for elective surgery. This diagnostic algorithm was adopted taking into consideration the high infection rate in Poland in the first quarter of 2020 and was in line with the guidelines of the Agency for Health Technology Assessment and Tariff System (AOTMiT), which limit the use of RT-PCR to high-risk patients (5).

Nevertheless, routine imaging studies, chest CT especially, in patients with low risk of infection remains quite controversial. Polish guidelines limit chest imaging only to patients with symptomatic COVID-19 $(5,6)$. Similarly, the Royal College of Radiologists does not recommend the routine preoperative chest CT scan at all. In asymptomatic patients with a positive RT-PCR result, the scan may be normal, whereas it can be falsely negative in $20 \%$ of symptomatic patients (7). On the other hand, when it is not possible to perform an RT-PCR test, a chest X-ray is the modality of choice in the diagnostics of a patient suspected of having COVID-19, particularly when other lung pathologies are suspected $(4,8,9)$. However, low sensitivity $(69 \%)$ makes chest X-rays poor in excluding COVID-19, while detecting infection must be supported and justified clinically (8-10).

The results of our study are in line with the abovementioned existing recommendations and confirm the necessity of clinical justification for chest imaging. Routine chest CT 
screening for COVID-19 changes in asymptomatic patients showed no benefits. It may be useful providing the clinical symptoms or past medical history suggest lung pathology, as shown by the higher rate of abnormal chest CT scans being observed in a group admitted in an emergency setting. Since a drop in lymphocyte count and increased leukocytes were not uncommon, A blood differential test has very limited value in COVID-19 screening. However, a blood smear may be considered helpful considering that lymphopenia is often present in patients with viral infections $(11,12)$.

In conclusion, the question of an optimal strategy for COVID-19 screening of patients admitted for elective surgery remains open. Laboratory blood tests, imaging and molecular COVID-19 diagnostic tests are all dedicated to symptomatic patients, which leaves asymptomatic patients as source of a potential horizontal transmission. Moreover, COVID-19 vaccination does not solve this problem as some patients remain unvaccinated or do not respond properly to vaccination. Laboratory blood tests such as blood smear may be helpful but are far from sufficient for an effective screening of potentially infected asymptomatic patients preoperatively. The need for an optimal diagnostic algorithm that would effectively limit the risk of SARS-CoV-2 transmission in medical centers is even more imperative in times of epidemiological stabilization.

\section{Conflicts of Interest}

All Authors declare they have no conflicts of interest regarding this article. The Authors received no financial support for the research or publication of this article.

\section{Authors' Contributions}

All Authors contributed equally to this work and approved the final version of the article. J. Cwalinski contributed to drafting of the article and prepared the literature review; A. Cwalinska conceived and designed the study; J. Paszkowski acquired the data and prepared the tables; T. Banasiewicz contributed critical revision of the article for important intellectual content; J. Herman prepared English language editing.

\section{References}

1 Green K, Allen AJ, Suklan J, Beyer FR, Price DA and Graziadio $\mathrm{S}$ : What is the role of imaging and biomarkers within the current testing strategy for the diagnosis of Covid-19? The Centre for Evidence-Based Medicine develops, promotes and disseminates better evidence for healthcare, April 8, 2020. Available at: https://www.cebm.net/covid-19/what-is-the-role-of-imaging-andbiomarkers-within-the-current-testing-strategy-for-the-diagnosisof-covid-19/ [Last accessed on September 23rd, 2021]

2 Liu Z, Zhang Y, Wang X, Zhang D, Diao D, Chandramohan K and Booth CM: Recommendations for surgery during the novel Coronavirus (COVID-19) epidemic. Indian J Surg: 1-5, 2020 PMID: 32292252. DOI: 10.1007/s12262-020-02173-3
3 Handbook of COVID-19 Prevention and Treatment. Chinese guidelines, 18/03/2020. Available at: https://www.alnap.org/system /files/content/resource/files/main/Handbook\%20of\%20COVID19\%20Prevention\%20and\%20Treatment.pdf [Last accessed on September 23rd, 2021]

4 Dennie C, Hague C, Lim RS, Manos D, Memauri BF, Nguyen ET and Taylor J: Canadian Society of Thoracic Radiology/Canadian Association of Radiologists consensus statement regarding chest imaging in suspected and confirmed COVID-19. Can Assoc Radiol J 71(4): 470-481, 2020. PMID: 32380844. DOI: 10.1177/08465371 20924606

5 Polish diagnostic, therapeutic and organizational recommendations for the care of individuals infected with SARS-CoV-2 or exposed to a SARS-CoV-2 infection. Available at: https://www.aotm.gov. pl/media/2020/07/Covid_FINAL-v-1.1-_wersja-EN-1.pdf [Last accessed on September 23rd, 2021]

6 Mitura K, Myśliwiec P, Rogula W, Solecki M, Furtak JP, Kazanowski M, Kłęk S, Nowakowski M, Pędziwiatr M, Zawadzki M, Wallner G and Sobocki J: Guidelines for the management of surgical departments in non-uniform hospitals during the COVID-19 pandemic. Pol Przegl Chir 92(2): 48-59, 2020. PMID: 32312919. DOI: 10.5604/01.3001.0014.1039

7 Statement on use of CT chest to screen for COVID-19 in preoperative patients. Available at: https://www.rcr.ac.uk/college/ coronavirus-covid-19-what-rcr-doing/clinical-information/statementuse-ct-chest-screen-covid [Last accessed on September 23rd, 2021]

8 Wong HYF, Lam HYS, Fong AH, Leung ST, Chin TW, Lo CSY, Lui MM, Lee JCY, Chiu KW, Chung TW, Lee EYP, Wan EYF, Hung IFN, Lam TPW, Kuo MD and Ng MY: Frequency and distribution of chest radiographic findings in patients positive for COVID-19. Radiology 296(2): E72-E78, 2020. PMID: 32216717. DOI: $10.1148 /$ radiol.2020201160

9 Hosseiny M, Kooraki S, Gholamrezanezhad A, Reddy S and Myers L: Radiology perspective of Coronavirus disease 2019 (COVID-19): Lessons from severe acute respiratory syndrome and Middle East respiratory syndrome. AJR Am J Roentgenol 214(5): 1078-1082, 2020. PMID: 32108495. DOI: 10.2214/AJR.20.22969

10 Jacobi A, Chung M, Bernheim A and Eber C: Portable chest Xray in coronavirus disease-19 (COVID-19): A pictorial review. Clin Imaging 64: 35-42, 2020. PMID: 32302927. DOI: 10.1016/j.clinimag.2020.04.001

11 Małecki P, Faltin K, Mania A, Mazur-Melewska K, Cwalińska A, Zawadzka A, Bukowska A, Lisowska K, Graniczna K and Figlerowicz M: Effects and safety of convalescent plasma administration in a group of Polish pediatric patients with COVID-19: A case series. Life (Basel) 11(3): 247, 2021. PMID: 33802763. DOI: 10.3390/life11030247

12 Mania A, Mazur-Melewska K, Lubarski K, Kuczma-Napierała J, Mazurek J, Jończyk-Potoczna K, Służewski W and Figlerowicz M: Wide spectrum of clinical picture of COVID-19 in children - From mild to severe disease. J Infect Public Health 14(3): 374-379, 2021. PMID: 33621800. DOI: 10.1016/j.jiph.2020.12.029

Received August 19, 2021

Revised September 21, 2021

Accepted September 24, 2021 